\title{
Direct Pore Matching for Fingerprint Recognition
}

\author{
Qijun Zhao, Lei Zhang, David Zhang, and Nan Luo \\ Biometrics Research Centre, Department of Computing, \\ The Hong Kong Polytechnic University, Kowloon, Hong Kong \\ \{csqj zhao, cslzhang, csdzhang, csnluo\} @ comp.polyu.edu.hk
}

\begin{abstract}
Sweat pores on fingerprints have proven to be useful features for personal identification. Several methods have been proposed for pore matching. The state-of-the-art method first matches minutiae on the fingerprints and then matches the pores based on the minutia matching results. A problem of such minutia-based pore matching method is that the pore matching is dependent on the minutia matching. Such dependency limits the pore matching performance and impairs the effectiveness of the fusion of minutia and pore match scores. In this paper, we propose a novel direct approach for matching fingerprint pores. It first determines the correspondences between pores based on their local features. It then uses the RANSAC (RANdom SAmple Consensus) algorithm to refine the pore correspondences obtained in the first step. A similarity score is finally calculated based on the pore matching results. The proposed pore matching method successfully avoids the dependency of pore matching on minutia matching results. Experiments have shown that the fingerprint recognition accuracy can be greatly improved by using the method proposed in this paper.
\end{abstract}

Keywords: Fingerprint recognition, pore matching, level-3 features, fusion.

\section{Introduction}

Automatic fingerprint recognition systems (AFRS), as well-known biometric techniques, are nowadays widely used in various applications such as forensics and access control [1-2]. Most existing AFRS utilize the minutiae (the endings and bifurcations of ridges, belonging to level-2 features) to recognize fingerprints and to identify persons [11-14]. However, as people's desire for higher security levels keeps increasing, it is highly necessary to base the recognition of fingerprints on more features, but not merely minutiae.

An example non-minutia feature is the sweat pores on fingerprints [3] as shown in Fig. 1. They have been used for a long time by forensic officers [4], and proven to be very distinctive features [10]. However, as level-3 fingerprint features, pores can not be reliably extracted from normal low-resolution fingerprint images [1]. Instead, a minimum of 1000dpi is required [3]. Recently, thanks to the development of high resolution imaging techniques, some pore-based AFRS have been proposed in literature [5-9].

In the earliest pore-based AFRS developed by Stosz and Alyea [5], fingerprints are first aligned by searching for the best alignment in a discretized transformation parameter space. The correlation between manually marked regions is used to choose the best alignment. Such correlation based method was later used by Kryszczuk et al. [6-7] 
in their study of using pores to recognize fragmentary fingerprints. After aligning the fingerprints, the pores on them can be matched by simply comparing their coordinates in the aligned fingerprint coordinate system. These pore matching methods are limited in the following factors. First, their accuracy highly depends on the way of discretizing the transformation parameters. Second, they have to search through all possible rotations and translations, which is computationally very expensive.

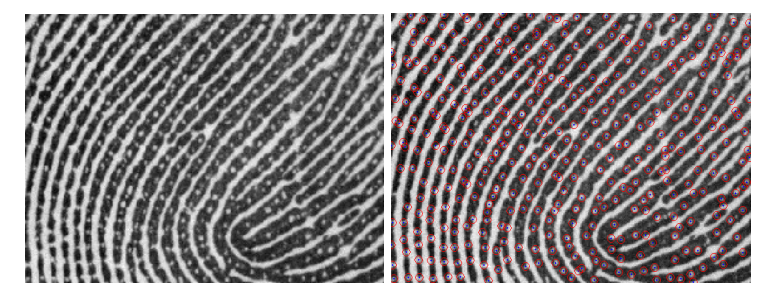

Fig. 1. Left: an example fingerprint image. Right: the extracted pores on it (marked by circles).

The state-of-the-art pore matching method was recently proposed by Jain et al. [8-9] In the method, the fingerprint images were first aligned based on the minutia features on them by using a string-matching algorithm. Minutiae on the fingerprints were then matched and paired. Pores lying in a rectangular neighborhood to each pair of matched minutiae were cropped and rotated according to the directions of the two minutiae. Afterwards, they were matched by using the iterative closest point (ICP) algorithm which is capable to handle sets of points with different numbers of points and can compensate for non-linear deformation between them. The average distance between matched pores was taken as the pore match score. This score was then fused with the minutia match score by using the weighted summation scheme. Compared with previous pore matching methods, this method can cope with fingerprint transformation more efficiently. However, it matches pores based on the minutia matching results. Consequently, the pore matching accuracy is limited by the minutia matching accuracy and the match scores of minutiae and pores will be not independent. Such dependency will impair the effectiveness of the subsequent fusion of the match scores.

In order to decouple the matching processes of minutiae and pores and to make the fusion of minutia and pore match scores more effective, this paper proposes a novel direct approach to matching pores on fingerprints. The proposed method incorporates the alignment into the matching process and can handle complicated transformations. It successfully avoids the dependency of pore matching on minutia matching. As a result, the fusion of the match scores of minutiae and pores is expected to be more effective because they are independent. Section 2 will introduce the proposed method in detail. Section 3 then gives our experimental results and section 4 concludes the paper.

\section{Direct Pore Matching}

In this paper, the pores are extracted from fingerprint images by using a state-of-theart method proposed by Zhao et al. [15] Fig. 1 gives an example fingerprint image and the pores extracted from it. The extracted pores are recorded by their locations on the 
fingerprint images and described by feature vectors defined later in this section. In order to match the pores on two fingerprint images, they are first pair-wise compared and initial correspondences between them are established based on their local features. The initial pore correspondences are then refined by using the RANSAC (RANdom SAmple Consensus) algorithm to give the final pore matching results. A pore match score is finally calculated for the two fingerprint images based on both the initial and final pore correspondences. Below, we introduce the proposed method step by step.

\subsection{Initial Pore Correspondence Establishment}

The first step of our pore matching approach is to pair-wise compare the pores and establish the initial correspondences between them. In order to fulfill this purpose, we have to associate each pore with a descriptor and present a method to compare the pores based on their descriptors. In the literature of computer vision, lots of local descriptors have been proposed to describe point features [16]. Most of them are based on the gradients in the local neighborhood to the points. Unfortunately, from our experiments, we observed that the gradients in the local neighborhood to pores on fingerprints are not very distinctive. In the context of fingerprint recognition, Jain et al. [17] proposed to use a bank of Gabor filters to generate a feature vector from the region surrounding the reference point to describe the fingerprint. However, this method failed to cope well with different transformations on fingerprints.

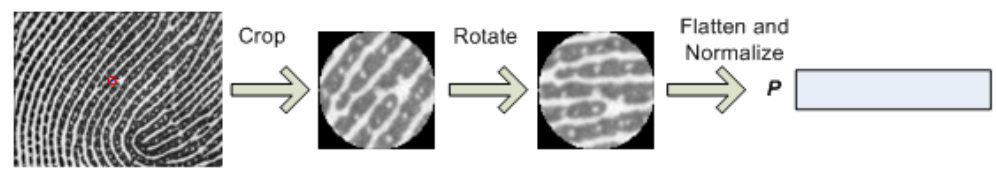

Fig. 2. The flowchart of constructing the descriptor for a pore (marked by circle). $P$ is a row vector whose entries are pixel values on the rotated circular neighborhood.

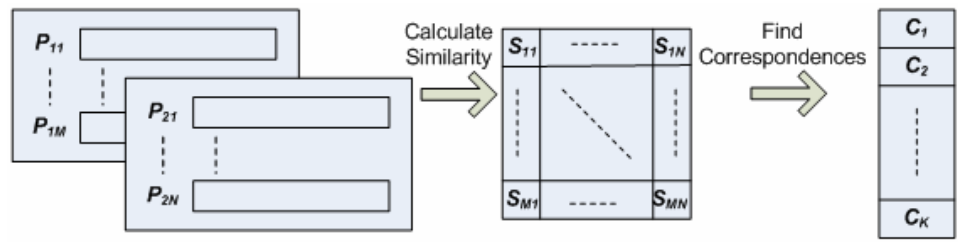

Fig. 3. The flowchart of establishing initial pore correspondences

Our descriptor in this paper is directly built from the pixel values in the local neighborhood to the pore. Specifically, we first smooth the fingerprint image with a Gaussian filter. This is to remove noise in the fingerprint. Then we set up a circular neighborhood to each pore. The circular neighborhood is rotated such that the ridge orientation at the location of the pore becomes horizontal. By using this rotated circular neighborhood, the descriptor is rotation invariant. A feature vector is then obtained by flattening the neighborhood and it is normalized to have zero mean and unit length. 
The normalization makes the feature vector invariant to monotone contrast changes and simplifies the subsequent computation of correlation between the feature vectors. This feature vector is defined as the descriptor for the pore. Fig. 2 shows the flowchart of the construction of the descriptor.

Given the descriptors for pores on two fingerprints, we can now compare them pair-wise and establish initial correspondences between them. Suppose $\left\{P_{1 i} \mid i=1,2, \cdots, M\right\}$ and $\left\{P_{2 j} \mid j=1,2, \cdots, N\right\}$ are the sets of pores on two fingerprints, $P_{1 i}, P_{2 j} \in R^{n}$ are the descriptors of the pores. We define the similarity between two pores $P_{i}$ and $P_{j}$ as

$$
S_{i j}=P_{i} P_{j}^{T},
$$

where ' $T$ ' denotes the transpose. Note that this similarity is equivalent to the correlation between the two feature vectors because they have zero mean and unit length after the normalization. After comparing all pores pair-wise, we get a similarity matrix $S \in R^{M \times N}$. We find for each pore on the first fingerprint a pore which is the most similar one to it among all the pores on the second fingerprint. Similarly, a pore is also found for each pore on the second fingerprint from the pores on the first fingerprint. If $P_{1 i_{k}}$ has its most similar pore as $P_{2 j_{k}}$ and $P_{1 i_{k}}$ is also the most similar pore of $P_{2 j_{k}}$, then a correspondence is established as $C_{k}=\left(P_{1 i_{k}}, P_{2 j_{k}}\right)$. Finally, we will get a number of pore correspondences. Let us assume there are $K$ pairs of corresponding pores and in the next subsection, we will further refine these correspondences to remove the false ones. Fig. 3 shows the flowchart of the initial pore correspondence establishment. An example result of initial pore correspondences determined on two fingerprint images is shown in Fig. 4.

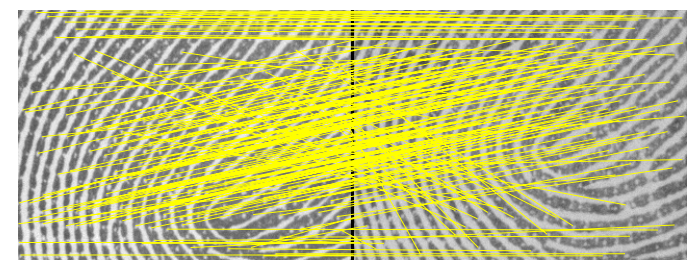

Fig. 4. The initial pore correspondences on two example fingerprint images

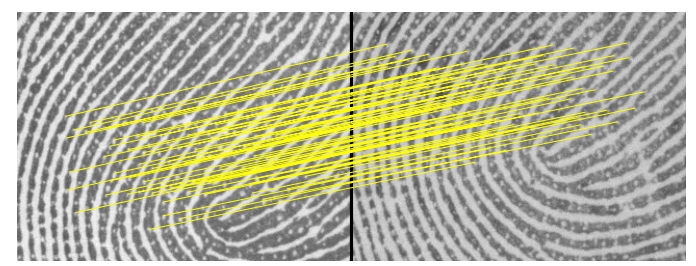

Fig. 5. The refined pore correspondences on the two example fingerprint images 


\subsection{Pore Correspondence Refinement}

In the above step of initial pore correspondence establishment, we consider only the local features and the translation and rotation on fingerprints. As a consequence, there are still lots of false pore correspondences among the initial pore correspondences as can be seen in Fig. 4. In this refinement step, we will cope with the pores in a global context and consider the non-rigid deformation between fingerprints.

Among all the pore correspondences, we call the correct correspondences as inliers and the false ones as outliers. These outliers can severely disturb the estimated transformation between fingerprints, and consequently make the matching results unreliable. In order to exclude the large number of outliers, we have to use a method which is robust to outliers. The ICP algorithm previously used for pore matching requires a good coarse alignment and is sensitive to outliers [20]. Therefore, we take the RANSAC algorithm proposed by Fishler and Bolles [18]. It is a widely used robust estimator for model fitting and has now become a standard in the field of computer vision. Based on the RANSAC algorithm, the pore correspondence refinement proceeds as follows.

First, we choose at random a set $C_{R}$ of three pairs of corresponding pores from the initial results. We choose three pairs because they are sufficient to exactly determine an affine transformation that we assume occurring to fingerprints. Let $(x, y)$ be a pore on the first fingerprint image and $(\tilde{x}, \tilde{y})$ its corresponding one on the second print after affine transformation. They are associated by

$$
\left(\begin{array}{l}
\tilde{x} \\
\tilde{y}
\end{array}\right)=\left[\begin{array}{ll}
a_{11} & a_{12} \\
a_{21} & a_{22}
\end{array}\right]\left(\begin{array}{l}
x \\
y
\end{array}\right)+\left[\begin{array}{l}
t_{x} \\
t_{y}
\end{array}\right] .
$$

There are six parameters in this model and therefore at least three pairs of corresponding pores are needed to uniquely determine the transformation. Based on the chosen three pairs of pores, we estimate the affine transformation simply by solving a set of linear equations.

Second, we evaluate the obtained transformation. The pores on the first fingerprint are transformed according to the obtained transformation. They are then compared with the pores on the second fingerprint. If the distance between two pores is below a given threshold, they are then thought to be matched. If two corresponding pores in the initial results are still matched after the transformation, the correspondence is then a correct one under the transformation. The set $C_{I}$ of these correct correspondences with respect to the transformation is the consensus set of the chosen correspondences and defines the inliers of $C_{R}$.

Third, we check the termination conditions. We set two conditions. One is that the maximum number of iterations $N_{m}$ has been reached and the other is that the sufficient number of iterations $N_{s}$ has been reached. $N_{m}$ is pre-specified, and $N_{s}$ is calculated as follows. Suppose we want to ensure with a probability $p$ that at least one chosen correspondence set $C_{R}$ in the iterations is free from outliers, in other words, all the correspondences and thus the transformation estimated based on them are correct. Let $\varepsilon$ be the percentage of outliers over the initial correspondence set with respect to 
the transformation obtained in the current iteration. According to [19], the sufficient number of iterations is then given by

$$
N_{s}=\log (1-p) / \log \left(1-(1-\varepsilon)^{3}\right) .
$$

If either of the two conditions is met, the algorithm is stopped and the largest set of inliers, $C_{I}^{*}$, is output as the final result of pore correspondences. Otherwise, go back to the first step and iterate until either of the termination conditions is met. Fig. 5 gives the result of refining the initial pore correspondences shown in Fig. 4. Obviously, the false matches are removed successfully.

\subsection{Match Score Computation}

In sub-sections 2.1 and 2.2, we show our approach to matching pores. In the process, we get the initial and refined correspondences between the pores on two fingerprints. Based on these results, we can define a similarity between the two fingerprints, i.e. the pore match score of the two fingerprints. In this paper, we define it as

$$
M S_{\text {pore }}=\frac{|C|+\left|C^{*}\right|}{2 \times \min \{M, N\}},
$$

where $C$ and $C^{*}$ are the set of initial pore correspondences and the set of refined pore correspondences respectively, ' $|C|$ ' denotes the number of elements in the set $C$, and $M$ and $N$ are the numbers of pores on the two fingerprints.

\section{Experiments}

In order to objectively assess the performance of the proposed method, we need a dataset of high resolution (at least 1000dpi) fingerprint images. Because there is no such a free high resolution live-scan fingerprint image database in the public domain, we built by ourselves a high resolution fingerprint scanner (approx. 1200dpi). Using this scanner, we collected a set of 1,480 fingerprint images of 148 fingers (see Fig. 1 for an example image). Five images were scanned for each finger in each of two sessions (about two weeks apart). These images have a spatial size of 320 by 240 pixels. They capture a fingerprint area of about $6.5 \mathrm{~mm}$ by $4.9 \mathrm{~mm}$. Therefore, they are very small partial fingerprints and it is very challenge to recognize such fingerprints. According to [6-7], the fingerprint recognition benefits more from the pores when the used fingerprint images cover small fingerprint areas. In order to emphasize the contribution of pores, we thus conducted our experiments in this paper with the above mentioned fingerprint images.

We compared our direct pore matching method (denoted as DP) with the state-of-theart minutia-based pore matching method (denoted as MICPP) proposed in [8]. In the implementation, the minutiae on two fingerprints are first extracted and matched by using the algorithm in [21]. After matching the minutiae, the pores lying in the neighborhoods of each pair of matched minutiae are matched by using the ICP algorithm, resulting in $N$ match scores ( $N$ is the number of pairs of matched minutiae), which are defined as the summation of two terms: the mean distance between all matched pores and the 
percentage of unmatched pores. The pore match score between the two fingerprints is finally defined as the average of the first three smallest match scores.

We consider the recognition accuracy in five cases: using only minutia match scores (denoted as MINU), using only pore match scores of DP, using only pore match scores of MICPP, using fused scores of MINU and DP (MINU-DP), and using fused scores of MINU and MICPP (MINU-MICPP). The fused score $M S$ is calculated by using the weighted summation scheme defined as

$$
M S=\omega \cdot M S_{\text {pore }}+(1-\omega) \cdot M S_{\text {minu }},
$$

where $M S_{\text {pore }}$ is the pore match score, $M S_{\text {minu }}$ is the minutia match score, and $\omega \in[0,1]$ is the weight for $M S_{\text {pore }}$. Before fusion, both the match scores of pores and minutiae are normalized to $[0,1]$ by using the maximum-minimum normalization.

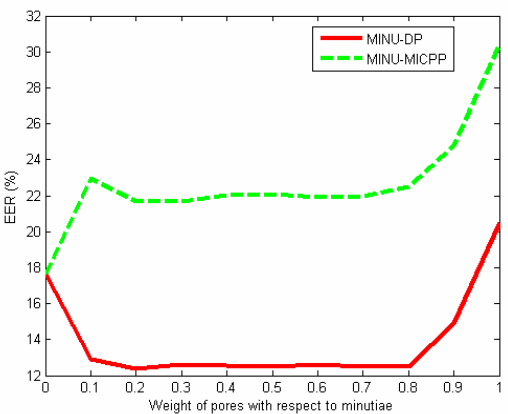

Fig. 6. The EER of MINU-DP and MINUMICPP when different weights are used

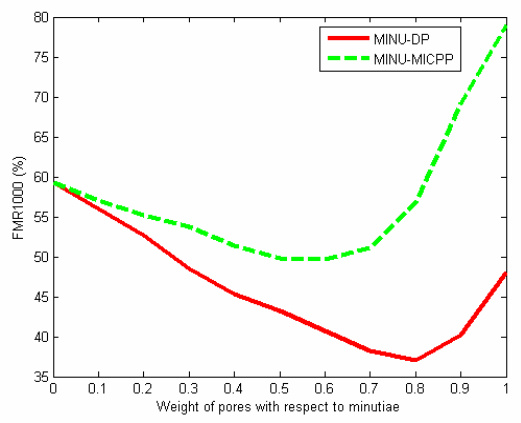

Fig. 7. The FMR 1000 of MINU-DP and MINU-MICPP when different weights are used

In the fingerprint recognition experiments, the following matches were carried out: 1) Genuine matches: Each of the fingerprint images in the second session was matched with all the fingerprint images in the first session, leading to 3,700 genuine matches, and 2) Imposter matches: the first fingerprint image of each finger in the second session was matched with the first fingerprint image of all the other fingers in the first session, resulting in 21,756 imposter matches. Based on these match scores, we calculated the equal error rates (EER) and FMR1000 [22] of the methods MINU-MICPP and MINU-DP when different weights were used. The results are presented in Figs. 6 and 7. As shown in Fig. 6, the EER of using only minutiae is $17.67 \%$, and the EER of the proposed DP and its counterpart MICPP are respectively $20.49 \%$ and $30.45 \%$. By fusing the minutia and pore match scores, our proposed method MINU-DP gets the lowest EER of $12.4 \%$ when $\omega=0.2$, which improves the EER of using only minutiae by $29.82 \%$. In contrast, the MINU-MICPP method fails to make any improvement at all. As for the FMR1000, according to the curves shown in Fig. 7, using only minutiae, the obtained FMR1000 is $59.24 \%$, whereas using only pores are $48.11 \%$ and $78.95 \%$ by DP and MICPP respectively. After fusing the minutia and pore match scores, the FMR1000 can be improved to $37.05 \%$ and $49.68 \%$ respectively by MINU-DP and MINU-MICPP. The best accuracy of each case is listed in Table 1. 
Table 1. The best EER and FMR1000 of the five cases

\begin{tabular}{c|c|c|c|c|c}
\hline Method & MINU & DP & MICPP & MINU-DP & MINU-MICPP \\
\hline EER & $17.67 \%$ & $20.49 \%$ & $30.45 \%$ & $12.4 \%$ & $21.66 \%$ \\
\hline FMR1000 & $59.24 \%$ & $48.11 \%$ & $78.95 \%$ & $37.05 \%$ & $49.68 \%$ \\
\hline
\end{tabular}

From these experimental results, we can see that when only using the pore match scores, the EER is worse than that of minutia match scores, but the FMR1000 could be better. This demonstrates that pores, if advanced pore matching methods are used, are good choices for applications where a very low false acceptance rate is required (or the cost of false acceptance is much higher than that of false rejection). When using only pores, our proposed method obtains much better recognition accuracy (32.71\% improvement on EER and 39.06\% improvement on FMR1000) compared with the MICPP method. When fusing the match scores of minutiae and pores, the accuracy can be further improved by our method. When the MICPP method is used for pore matching, compared with the results of using only minutiae, no improvement is observed on EER and 16.14\% improvement on FMR1000. But when using our proposed method, the fusion can achieve $29.82 \%$ and $37.46 \%$ improvement respectively upon the EER and FMR1000 of using only minutia match scores. We believe that this improvement owes to the following factors. First, our proposed direct pore matching method incorporates the alignment into the matching process and can better cope with complicated transformations between fingerprints. Second and more important, it enables the independency between minutia and pore matching processes and thus makes the fusion between the minutia and pore match scores more effective.

\section{Conclusions}

This paper presents a direct pore matching approach for fingerprint recognition. It incorporates the alignment into the pore matching process and can cope with complicated transformations between fingerprints. By making the pore matching independent from the minutia matching, it enables the fusion of the minutia and pore match scores more effective in improving the fingerprint recognition accuracy. Experiments testified that better recognition accuracy can be obtained by using the proposed method. Compared with the state-of-the-art minutia-based pore matching method, our method can achieve more than $30 \%$ improvement on the recognition accuracy when using only pores. By fusing minutia and pore match scores, our method improves the recognition accuracy with respect to that of using only minutiae by $29.82 \%$ and $37.46 \%$ respectively in terms of EER and FMR1000, whereas the counterpart method makes no improvement at all on EER and only $16.14 \%$ improvement on FMR1000. A shortcoming of the proposed method is its complexity in describing the pores. Considering the large number of pores on fingerprints, it is worthy to reduce the dimension of the pore descriptors while not impairing the discrimination power and to speed up the comparison of pores.

\section{Acknowledgements}

The work is partially supported by the CERG fund from the HKSAR Government, the central fund from The Hong Kong Polytechnic University, the Natural Science 
Foundation of China (NSFC) under Contract No. 60620160097, and the National High-Tech Research and Development Plan of China (863) under Contract No. 2006AA01Z193 and 2007AA01Z195.

\section{References}

1. Maltoni, D., Maio, D., Jain, A.K., Prabhakar, S.: Handbook of Fingerprint Recognition. Springer, New York (2003)

2. Ratha, N., Bolle, R.: Automatic Fingerprint Recognition Systems. Springer, New York (2004)

3. CDEFFS: Data Format for the Interchange of Extended Fingerprint and Palmprint Features, Working Draft Version 0.2 (2008), http://fingerprint.nist.gov/standard/cdeffs/index.html

4. Ashbaugh, D.R.: Quantitative-Qualitative Friction Ridge Analysis: An Introduction to Basic and Advanced Ridgeology. CRC Press, Boca Raton (1999)

5. Stosz, J.D., Alyea, L.A.: Automated System for Fingerprint Authentication Using Pores and Ridge Structure. In: SPIE Conference on Automatic Systems for the Identification and Inspection of Humans, vol. 2277, pp. 210-223 (1994)

6. Kryszczuk, K., Drygajlo, A., Morier, P.: Extraction of Level 2 and Level 3 Features for Fragmentary Fingerprints. In: Second COST Action 275 Workshop, pp. 83-88 (2004)

7. Kryszczuk, K., Morier, P., Drygajlo, A.: Study of the Distinctiveness of Level 2 and Level 3 Features in Fragmentary Fingerprint Comparison. In: Maltoni, D., Jain, A.K. (eds.) BioAW 2004. LNCS, vol. 3087, pp. 124-133. Springer, Heidelberg (2004)

8. Jain, A.K., Chen, Y., Demirkus, M.: Pores and Ridges: Fingerprint Matching Using Level 3 Features. In: 18th International Conference on Pattern Recognition, vol. 4, pp. 477-480 (2006)

9. Jain, A.K., Chen, Y., Demirkus, M.: Pores and Ridges: Fingerprint Matching Using Level 3 Features. IEEE Trans. Pattern Analysis and Machine Intelligence 29(1), 15-27 (2007)

10. Roddy, A., Stosz, J.: Fingerprint Features - Statistical Analysis and System Performance Estimates. Proceedings of the IEEE 85(9), 1390-1421 (1997)

11. Maio, D., Maltoni, D.: Direct Gray-Scale Minutiae Detection in Fingerprints. IEEE Trans. Pattern Analysis and Machine Intelligence 19(1), 27-40 (1997)

12. Jiang, X., Yau, W.Y., Ser, W.: Minutiae Extraction by Adaptive Tracing the Gray Level Ridge of the Fingerprint Image. In: ICIP 1999, vol. 2, pp. 852-856 (1999)

13. Chen, X., Tian, J., Cheng, J., Yang, X.: Segmentation of Fingerprint Images Using Linear Classifier. EURASIP Journal on Applied Signal Processing 2004(4), 480-494 (2004)

14. Wu, C., Tulyakov, S., Govindaraju, V.: Robust Point-Based Feature Fingerprint Segmentation Algorithm. In: Lee, S.-W., Li, S.Z. (eds.) ICB 2007. LNCS, vol. 4642, pp. 1095-1103. Springer, Heidelberg (2007)

15. Zhao, Q., Zhang, L., Zhang, D., Luo, N., Bao, J.: Adaptive Pore Model for Fingerprint Pore Extraction. In: ICPR 2008 (2008)

16. Mikolajczyk, K., Schmid, C.: A Performance Evaluation of Local Descriptors. IEEE Trans. Pattern Analysis and Machine Intelligence 27(10), 1615-1630 (2005)

17. Jain, A.K., Prabhakar, S., Hong, L., Pankanti, S.: Filterbank-based Fingerprint Matching. IEEE Trans. Image Processing 9(5), 846-859 (2000)

18. Fishler, M., Bolles, R.: Random Sample Consensus: A Paradigm for Model Fitting with Applications to Image Analysis and Automated Cartography. Comm. ACM 24(6), 381395 (1981) 
19. Hartley, R., Zisserman, A.: Multiple View Geometry in Computer Vision, 2nd edn. Cambridge Univ., Cambridge (2003)

20. Phillips, J.M., Liu, R., Tomasi, C.: Outlier Robust ICP for Minimizing Fractional RMSD. In: 6th International Conference on 3-D Digital Imaging and Modeling, pp. 427-434 (2007)

21. Feng, J.: Combining Minutiae Descriptors for Fingerprint Matching. Pattern Recognition 41, 342-352 (2008)

22. Maio, D., Maltoni, D., Cappelli, R., Wayman, J.L., Jain, A.K.: FVC 2002: Second Fingerprint Verification Competition. In: ICPR 2002, pp. 811-814 (2002) 